bridging therapy. Subsequently, according to ACR guidelines, a therapy with TNF-alpha inhibitor, adalimumab, was initiated. Within a month, a substantial reduction of the low back pain was noted, with a decrease in juvenile spondyloarthritis disease activity score (jSpADA).

Low back pain often begins in childhood, with the prevalence in adolescence being similar to that in adulthood. Among many possible causes, inflammatory etiology should be thoroughly considered due to irreversible damage if not treated adequately. Therefore, children and adolescents complaining of a back pain, especially in the presence of other signs of arthritis, should be referred to pediatric rheumatologist for a further work-up and treatment.

\section{THE ART OF RECOGNIZING PAIN AMPLIFICATION SYNDROME IN CHILDREN}

${ }^{1}$ Vana Vukić*, ${ }^{2}$ Mandica Vidović, ${ }^{1,2}$ Miroslav Harjaček, ${ }^{1,2}$ Lovro Lamot. ${ }^{1}$ Institution of the presenting author; ${ }^{2}$ School of Medicine University of Zagreb;, Department of clinical immunology and rheumatology, Department of Pediatrics, University Hospital Centre Sestre milosrdnice, Zagreb, Croatia

\subsection{6/archdischild-2021-europaediatrics.449}

Juvenile fibromyalgia is a pain amplification syndrome characterized by diffuse idiopathic musculoskeletal pain, most frequently affecting children older than 10 years. Girls are more commonly affected than boys, with the ratio of $1: 4-8$. American College of Rheumatology criteria for diagnosis include absence of other disorder that could explain the pain, symptoms lasting for $\geq 3$ months, widespread pain index (WPI) $\geq 7$ and symptom severity score (SSS) $\geq 5$ or WPI $3-6$ and SSS $\geq 9$.

We present a case of 15 years old girl with diffuse and persistent pain along with episodes of impaired consciousness. Those episodes occurred once a month for the last 3 years and were characterized by collapse and/or inability to move or speak for several minutes. They were preceded by paraesthesia of face, arms and feet, but have never resulted in injury. In addition, patient complained of pain in radiocarpal joints, hips, low back, groins and neck, along with extreme tiredness, poor sleeping and inability to concentrate.

Extensive examination was undertaken. The neurological assessment included brain MRI and EEG, both of which were normal. The rheumatologic evaluation revealed multiple painful points with WPI 9 and SSS 8. The patient didn't experience morning stiffness, joint swelling or fever. Extensive laboratory workup and MRI of sacroiliac joints along with a lumbar and thoracic portion of the spine excluded the inflammatory aetiology. The psychological assessment revealed poor stress coping strategies. The patient was diagnosed with fibromyalgia and conversion disorder manifested in the form of pseudoseizures. Physical therapy and psychotherapy were advised, along with melatonin for better sleep regulation, which led to a mild improvement of symptoms within a month.

Fibromyalgia and diverse psychological distress are causally related. Accordingly, conversion symptoms are not uncommon. Therefore, a multidisciplinary team approach is necessary when caring for these patients, in addition to providing treatment not only for the affected individual but for the entire family as well.

\section{KAWASAKI DISEASE WITH PULMONARY MANIFESTATIONS - A CASE REPORT OF THREE PATIENTS}

${ }^{1}$ Ivana Sosa Filjak*, ${ }^{2}$ Maja Batinica, ${ }^{2,3}$ Arnes Rešić, ${ }^{2,4}$ Alenka Gagro. ${ }^{1}$ Institution of the presenting author; 'General Hospital Zabok; ' ${ }^{2}$ Clinic for Pediatrics, Clinic for Children's Diseases Zagreb, Faculty of Medicine, University of Zagreb; ${ }^{3}$ Faculty of Medicine, University of Split; ${ }^{4}$ Faculty of Medicine, Josip Juraj Strossmayer University of Osijek

\subsection{6/archdischild-2021-europaediatrics.450}

Kawasaki disease (KD) represents systematic vasculitis of unknown etiology that typically occurs in childhood. Its most important complications are coronary artery changes described in 15 to $25 \%$ of untreated children. The diagnosis is based on fever criteria longer than five days and at least four additional criteria: polymorphic exanthema, changes in the extremities, mucosal changes in the oral cavity or on the lips, bilateral conjunctival injection and unilateral cervical lymphadenopathy. Affection of lungs is rare manifestation in $\mathrm{KD}$ and can present as pneumonia, pulmonary nodules, bronchopneumonia, hydropneumothorax and pleural effusion. During a ten year period, we treated 11 children diagnosed with KD in our clinic, of which $\mathrm{KB}$ presented with clinical and image-proven lung involvement during the acute phase.

CASE REPORT: Patient 1.: A 16 year old adolescent presented with persistent fever, cough, macular rash of the trunk and extremities, conjunctival injection and hepatosplenomegaly. Bilateral interstitial pneumonia with bilateral pleural effusion was radiologically confirmed. An MSCT of thorax was performed, in which nodular confluent consolidations and ground glass opacities, along with pleural effusion, were described. No cardiological manifestations occurred during acute phase or during follow up of the patient.

Patient 2.: A 6 year old boy presented with fever, abdominal pain, haemorrhagic diarrhoea, oedema of the eyelids, feet and hands, conjunctival injection and maculo-papular rash in the lower abdomen. Radiological and ultrasound findings showed alveolo-interstitial pneumonia with bilateral pleural effusion. Cardiological processing proved myocarditis, fusiform aneurysm of the right coronary artery and dilatation of the left coronary artery.

Patient 3.: A 5 year old girl presented with prolongated fever, inguinal lymphadenitis, oedema of the eyelids and lips, conjunctival injection, macular rash on the trunk and extremities and erythema multiforme on the abdomen. Chest x-ray and ultrasound verified right-sided supradiaphragmatic infiltration with minor bilateral pleural effusion. The course od the disease was complicated by multiorgan failure syndrome and the development of $\mathrm{KD}$ associated shock syndrome. Echocardiography verified changes on both coronary arteries.

Microbiological analysis was negative in all patients with $\mathrm{KB}$ and pulmonary manifestations. Pulmonary manifestations did not improve upon administration of antibiotic treatment. All patients answered positive to treatment with intravenous immunoglobulins and acetylsalicylic acid. Corticosteroids and additional intravenous immunoglobulins were administered in patient with KD associated shock.

Conclusion According to the literature, pulmonary manifestations have been described in 20 patients (1). Of these, seven had coronary artery changes, 10 patients had incomplete disease and seven patients requested an additional, second dose of intravenous immunoglobulins with or without corticosteroids. Although the etiology of pulmonary manifestations in 
$\mathrm{KD}$ is currently unknown, according to the available literature and our experiences, it is important to point out that therapy for $\mathrm{KD}$ also leads to the resolution of lung problems and findings.

\section{ASSOCIATION BETWEEN GASTROINTESTINAL MANIFESTATIONS AND THE RISK OF RENAL DISEASE IN CHILDREN WITH IGA VASCULITIS}

\begin{abstract}
${ }^{1}$ Mario Sestan*, ${ }^{1}$ Nastasia Kifer, ${ }^{2}$ Sasa Srsen, ${ }^{3}$ Aleksandar Ovuka, ${ }^{4}$ Mateja Batnozic Varga, ${ }^{4}$ Matej Sapina, ${ }^{1}$ Martina Held, ${ }^{1}$ Maja Ban, ${ }^{1}$ Ana Kozmar, ${ }^{1}$ Marijana Coric, ${ }^{1}$ Stela Bulimbasic, ${ }^{1}$ Kristina Crkvenac, ${ }^{1}$ Danko Milosevic, ${ }^{1}$ Marijan Frkovic, ${ }^{5}$ Alenka Gagro, ${ }^{1}$ Marija Jelusic. ${ }^{1}$ University Hospital Centre Zagreb, University of Zagreb, School of Medicine, Zagreb; ${ }^{2}$ University Hospital Centre Split, University of Split, School of Medicine, Split; ${ }^{3}$ University Hospital Centre Rijeka, University of Rijeka, School of Medicine, Rijeka; ${ }^{4}$ University Hospital Centre Osijek, University of Osijek, School of Medicine, Osijek; ${ }^{5}$ Children's Hospital Zagreb, Zagreb; University of Osijek, School of Medicine, Osijek, Croatia
\end{abstract}

\subsection{6/archdischild-2021-europaediatrics.451}

Indroduction $\operatorname{IgA}$ vasculitis (IgAV) is the most common childhood-vasculitis in which more than $50 \%$ of children develop gastrointestinal (GI) symptoms. In 10-20\% of patients serious complications such as intussusception, bowel perforation, and massive bleeding may occur. The most important complication is the development of nephritis with progression to chronic renal failure in about 3\% of children. The aim of the research was to analyze clinical and biochemical parameters in patients with IgAV and GI manifestations.

Methods This retrospective study included children with IgAV reviewed in five Croatian University Centers for pediatric rheumatology in the period 2009 to 2019.

Results Out of 611 children with IgAV, 320 were males and 291 were females. The overall GI symptoms prevalence was $45.9 \%$ and the median (range) age at diagnosis was 6.42 (4.58.83) years. Among patients with GI symptoms there were 1.44 times more males $(\mathrm{N}=166)$ than females $(\mathrm{N}=115)$, which was statistically significant $(\mathrm{p}=0.003)$. Patients with GI symptoms had less infections before the appearance of purpura $(59.8 \%$ vs. $70.9 \%, p=0.005)$ and were found to be significantly more likely to have rash distributed on the trunk $(61.9 \%$ vs. $48.5 \%, p=0.001)$, and upper extremities $(35.2 \%$ vs. $24.7 \%, \mathrm{p}=0.006)$, as well as generalized rash $(38.8 \%$ vs. $28.3 \%, \mathrm{p}=0.008)$. These patients also had significantly higher values of C-reactive protein, leukocyte count, erythrocytes and platelets, hemoglobin, hematocrit and D-dimer concentrations and lower levels of $\operatorname{IgG}$ and IgM. In our cohort 42 out of 281 children (14.9\%) had the most severe GI manifestations (intussusception and/or massive GI bleeding) with significantly higher values of 24-hour urine protein levels and D-dimer concentrations and lower total serum protein, albumin, IgG, IgM and C3 levels in comparison with children whose GI manifestations were not severe. Predictors of severe GI involvement were: relapse of the disease, generalized rash, rash extended on upper extremities, rash extended to the face, recurrent rash and renal involvement, as well as lower values of prothrombin time, fibrinogen and IgM among the laboratory parameters. Patients with GI symptoms were 1.68 times more likely to develop nephritis, and this probability was 2.58 times higher if GI symptoms occurred before other symptoms. Other predictors of nephritis were: severe and moderate GI manifestations, recurrent rash, one or more relapses of IgAV, and older age.

Conclusion Older children with IgAV and severe GI manifestations in whom IgAV begun with GI symptoms had a higher risk of acute and chronic complications of the disease.

SUPPORT: Croatian Science Foundation project IP-2019-048822

\section{THE IMPACT OF SYSTEMIC IMMUNOMODULATORY THERAPY ON THE INTRAOCULAR INFLAMMATION AND THE NEED FOR TOPICAL GLUCOCORTICOID THERAPY IN PATIENTS WITH JUVENILE IDIOPATHIC ARTHRITIS- ASSOCIATED UVEITIS} Martina Galiot Delic, 'Sonja Jandrokovic, ${ }^{2}$ Martina Held, ${ }^{2}$ Marijan Frkovic, ${ }^{2}$ Marija Jelusic, ${ }^{1}$ Nenad Vukojevic. 'University Hospital Centre Zagreb, Department of Ophthalmology, University of Zagreb School of Medicine; ${ }^{2}$ University Hospital Centre Zagreb, Department of Pediatrics, University of Zagreb School of Medicine, Zagreb, Croatia

10.1136/archdischild-2021-europaediatrics.452

Indroduction Juvenile idiopathic arthritis associated uveitis (JIA-U) is the most common and potentially most destructive extraarticular manifestation of JIA. The aim of this research was to determine the need for topical glucocorticoid therapy (TGC) in patients with JIA-U on systemic biological therapy in comparison to patients treated with methotrexate (MTX) only.

Methods We have conducted a longitudinal observational study with JIA-U patients in whom systemic immunomodulatory treatment (IMT: biologics and/or MTX) was introduced and who were followed at least 3 months in the period between 2011 and 2017. The data about the number of cells in the anterior chamber (AC) according to Standardization of Uveitis Nomenclature (SUN) Working Group criteria, related to TGC, systemic therapy and JIA complications were collected during each examination. Generalized linear mixed models were used to analyze the relationships between treatment with biologics, MTX, TGC and the grade of inflammation in AC according to SUN criteria.

Results 38 JIA-U patients (69 eyes) with median (range) age of 4.9 (2-15) years and follow up period of 209 (19-381) weeks were included. There were a total of 1205 examinations. At the first examination JIA-U was detected in 16 $(42.1 \%)$ of patients, $59(79.7 \%)$ of the eyes had $\leq 1+$ cells in the $\mathrm{AC}$, and in $19(50 \%)$ of JIA-U patients complications were already present. MTX was introduced in $23(60.5 \%)$ JIA-U patients before the initiation of the study. $8(21 \%)$ had already received biologics, while in $4(10.5 \%)$ prior systemic glucocorticoids were also used. Before the completion of the study, all patients received MTX and 40\% JIA-U were treated with biologics. The average number of TGC doses decreased from 3.74 at baseline to 0.72 in the 48th month. After Friedman and the post hoc test a significant difference in the daily doses of TGC could be seen from the 12th month after application of systemic IMT. The number of daily doses of TGC as well as the degree of inflammation in AC per eye decreased over time. Using generalized linear mixed models it was shown that the treatment with biologics, but not with MTX and systemic glucocorticoids, was associated with lower 